\title{
Strongly Absolute Stability Problem of Descriptor Systems
}

\author{
Chunyu YANG, Qingling ZHANG, Linna ZHOU \\ Institute of Systems Science, Northeastern University \\ Shenyang, Liaoning province, 110004, P.R. China \\ e-mail:qlzhang@mail.neu.edu.cn
}

Received: May 2006

\begin{abstract}
This paper considers Lur'e type descriptor systems (LDS). The concept of strongly absolute stability is defined for LDS and such a notion is a generalization of absolute stability for Lur'e type standard state-space systems (LSS). A reduced-order LSS is obtained by a standard coordinate transformation and it is shown that the strongly absolute stability of the LDS is equivalent to the absolute stability of the reduced-order LSS. By a generalized Lyapunov function, we derive an LMIs based strongly absolute stability criterion. Furthermore, we present the frequency-domain interpretation of the criterion, which shows that the criterion is a generalization of the classical circle criterion. Finally, numerical examples are given to illustrate the effectiveness of the obtained results.
\end{abstract}

Key words: Lur'e type systems, descriptor systems, strongly absolute stability, linear matrix inequality (LMI).

\section{Introduction}

In the last two decades, descriptor systems have been one of the major research fields of control theory due to their comprehensive applications in the Leontief dynamic model (Silva and De Lima, 2003), electrical and mechanical models (Campbell, 1980; Muller, 1997), etc. Depending on the applicable areas, these models are also called singular systems, semi-state systems, differential-algebraic systems, or generalized state-space systems. As to the stability of linear time-invariant descriptor systems, many sufficient and necessary conditions have been reported (Lewis, 1986; Dai, 1989; Ishihara and Terra, 2002) and almost all of these results are expressed by matrix rank conditions and matrix inequality which can be verified efficiently by the existing tools. However, stability problem of nonlinear descriptor systems has not been thoroughly investigated though there are some preliminary results. In (Vladimir, 1986; Vladimir, 1987) and (Vladimir and Mirko, 1987), the researchers investigate the stability of nonlinear descriptor systems under the assumption that the set of consistent initial conditions is given. In (Wu and Mizukami, 1995), the Lyapunov stability theory for standard state-space systems is extended to nonlinear descriptor systems. In (Wu et al., 2002), the authors present a sufficient condition for the system to be locally asymptotically stable. As stated in ( $\mathrm{Li}$ and 
Liu, 1998), there are several difficulties in the study of stability problem for nonlinear descriptor systems: (i) it is not easy to satisfy conditions of the existence and uniqueness of solutions; (ii) there often exist impulses and jumps in the solutions; (iii) it is difficult to calculate the derivatives of Lyapunov functions along the solutions.

In 1944, Lur'e and Postnikov introduced a novel method to deal with stability problem of nonlinear systems, which is called "nonlinearities isolation method" later and has been developed as the absolute stability theory. For many practical control systems, by using this method, the nonlinear characteristic can be separated, which results in a feedback system called Lur'e type system whose forward path is a linear time-invariant system and the feedback path is a nonlinearity with sector constraints (Mohler, 1991). Lur'e type standard state-space systems (LSS) have been widely investigated and the most celebrated ones are the Popov criterion(PC) and circle criterion(CC) (Haddad and Bernstein, 1993; Haddad and Bernstein, 1994). The PC is less conservative than CC because the Lyapunov function used by PC is a Lur'e type Lyapunov function which explicitly depends on the nonlinearity, while CC is related to a quadratic Lyapunov function. And the $\mathrm{CC}$ can deal with more diverse nonlinearities including time-varying ones. However, investigation on Lur'e type descriptor systems(LDS) is very few. In (Lee and Chen, 2003), an LMI based strictly positive real(SPR) lemma is given for discrete-time descriptor systems. Under the admissibility and SPR assumption of the involved linear time-invariant descriptor systems, it shows that the globally asymptotic stability of the feedback connection is guaranteed for the whole class of memoryless time-varying nonlinearities with dynamics constrained in the first and third quadrants. But it does not consider the impulsive behavior of the overall system.

In this paper, we investigate the stability of LDS. First, the notion of index of nonlinear descriptor systems is recalled and discussed. For convenience and without any confusion, an index one nonlinear descriptor system is called to be impulsive-free in this paper. Subsequently, strongly absolute stability of LDS is defined to be globally asymptotically stable and impulsive-free. Such a concept is a generalization of the absolute stability of LSS as well as the admissibility of linear time-invariant descriptor systems. Then, it is shown that the admissibility of the linear part is a necessary condition for the strongly absolute stability of the LDS. Consequently, under the assumption that the linear part of the LDS is admissible, by the standard coordinate transformation, a reduced-order LSS is obtained. Whereafter, an LMIs based stability criterion is derived by a generalized Lyaponov function and S-procedure. Furthermore, we present the frequency-domain interpretation of the LMIs based stability criterion, which shows that the criterion is a generalization of the well known circle criterion. Finally, two numerical examples illustrate the effectiveness of our results.

\section{Preliminaries and Basic Results}

The notations that are used here are standard in most respects. We use $R$ to denote the set of real numbers and $C$ to denote the complex plane. $R^{n}$ and $R^{n_{1} \times n_{2}}$ are the obvious extensions to vectors and matrices of the specified dimensions. Let $I$ or $I_{r}$ denote 
the identity matrix with appropriate dimension. $M$ is a matrix with proper dimension, $M^{T}$ and $M^{H}$ stand for the transpose and complex conjugate transpose of $M$, respectively. $\operatorname{Re}(\cdot)$ and $\operatorname{Im}(\cdot)$ denote the real part and the image part of a complex number, respectively.

Consider a linear time-invariant descriptor system

$$
\begin{aligned}
& E \dot{x}=A x+B \omega, \\
& y=C x+D \omega
\end{aligned}
$$

where $x \in R^{n}$ is the state variable, $\omega$ is the input variable, the matrices $A, E \in$ $R^{n \times n}, B \in R^{n \times m}, C \in R^{m \times n}, D \in R^{m \times m}, \operatorname{rank}(E)=r<=n$.

First, we state here some basic definitions which will be used in the sequel and can be founded in (Campbell, 1980; Dai, 1989). If $\operatorname{det}(s E-A) \neq 0$ for some complex number $s$, then the pair $(E, A)$ is said to be regular. A regular pair $(E, A)$ is called impulsive-free if $\operatorname{deg} \operatorname{det}(s E-A)=\operatorname{rank} E$. Note that an impulsive-free pair $(E, A)$ is implied to be regular. If all roots of $\operatorname{det}(s E-A)=0$ lie in $\operatorname{Re}(s)<0,(E, A)$ is called stable. And the pair $(E, A)$ is called admissible if it is impulsive-free and stable. It is proved in (Lewis, $1986)$ that $(E, A)$ is regular if and only if there exist two nonsingular matrices $M$ and $N$ such that $(E, A)$ can be transformed to the Weierstrass canonical form

$$
M E N=\left[\begin{array}{cc}
I_{r} & 0 \\
0 & J
\end{array}\right], \quad M A N=\left[\begin{array}{cc}
A_{1} & 0 \\
0 & I
\end{array}\right],
$$

where $J \in R^{(n-r) \times(n-r)}$ is a nilpotent matrix, $A_{1} \in R^{r \times r}$. And $\operatorname{system}(E, A)$ is impulsive-free if and only if $J=0$.

Definition 1 (Sun et al., 1994; Zhang et al., 2002). Let $G(s)=C(s E-A)^{-1} B+D$, then

1) $G(s)$ is said to be positive real (PR) if $G(s)$ is analytic in $\operatorname{Re}(s)>0$ and satisfies $G(s)+G^{*}(s)>=0$ for $\operatorname{Re}(s)>0$.

2) $G(s)$ is said to be strictly positive real (SPR) if $G(s)$ is analytic in $\operatorname{Re}(s)>=0$ and satisfies $G(j \omega)+G^{*}(j \omega)>0$ for $\omega \in[0,+\infty)$.

3) $G(s)$ is said to be extended strictly positive real (ESPR) if it is SPR and satisfies $G(j \infty)+G^{*}(j \infty)>0$.

Consider the following nonlinear descriptor system

$$
E \dot{x}=F(x, t)
$$

where $F: R^{n} \times\left[t_{0},+\infty\right) \rightarrow R^{n}$ is smooth enough and $F(0, t) \equiv 0, \forall t>=t_{0}$.

DEFINITION 2 (Brenan et al., 1996). System (3) is said to be of index one if the constant coefficient system

$$
E \dot{w}-F_{x}(\hat{x}, \hat{t}) w=g(t)
$$


is impulsive-free for all $(\hat{x}, \hat{t})$ in a neighborhood of the graph of the solution, where $F_{x}$ is the Jacobian matrix $\partial F / \partial x$.

REMARK 1. The notion of index plays a key role in the classification and behavior of nonlinear descriptor systems and can be thought of as the generalization of the nilpotent index of a linear time-invariant descriptor system (Brenan et al., 1996). Furthermore, considering (2), the nilpotent index of system (1) is actually the nilpotent index of matrix $J$. So, system (1) is impulsive-free if and only if it is of index one. Thus, for convenience, it is reasonable to call system (3) to be impulsive-free if it is of index one. From the implicit function, the solvability of a impulsive-free system (3) is easy to guarantee (Brenan et al., 1996).

\section{Strongly Absolute Stability}

Consider the following Lur'e type descriptor system

$$
\begin{aligned}
& E \dot{x}=A x+B \omega, \\
& \sigma=C x+D \omega, \\
& \omega=-\phi(\sigma),
\end{aligned}
$$

where $\phi(\sigma)$ is assumed to be a time-invariant smooth enough nonlinear function.

We call $\phi(\cdot) \in F[0, K]$ if $\phi(0)=0$ and satisfy the following sector constraint

$$
\phi^{T} \phi<=\phi^{T} K \sigma
$$

where $K$ is a symmetric positive definite matrix.

In the sequel, we suppose the following.

Assumption 1. LDS (5) is well-posed, that is, identity

$$
\omega=-\phi(C x+D \omega)
$$

has a unique solution for every $x$ in the domain of interest.

Assumption 2. $(E, A)$ is admissible.

REMARK 2. Assumption 1 is a routine for the discussion of robust stability problem (Khalil, 1996). And Assumption 2 is a necessary condition for strongly absolute stability of LDS (5), which will be shown later.

If $E=I$, LDS (5) reduces to a LSS that has been widely studied. Next, the absolute stability of LSS is extended to LDS.

DEFINITION 3. LDS (5) is said to be strongly absolutely stable with respect to $F[0, K]$, if for $\forall \phi \in F[0, K]$, LDS (5) is globally asymptotically stable and impulsive-free. 
REMARK 3. It is easy to see that Definition 3 is a generalization of absolute stability of LSS as well as admissibility of linear time-invariant descriptor systems. So it is different from the notion of absolute stability given in (Lee and Chen, 2003) which only considers the global stability of descriptor systems.

If we set $\phi(\sigma)=K_{\Delta} \sigma$, where $K_{\Delta}$ is arbitrary symmetric matrix with $0<=K_{\Delta}<=$ $K$, LDS (5) reduces to a linear time-invariant descriptor system

$$
E \dot{x}=\left(A-B\left(I+D K_{\Delta}\right)^{-1} K_{\Delta} C\right) x
$$

which is called the linearized system of LDS (5). Note that $\left(I+D K_{\Delta}\right)^{-1}$ does exist since LDS (5) is well-posed.

From Definition 3 and Remark 3, the following result is obvious.

Theorem 1. LDS (5) is strongly absolutely stable with respect to $F[0, K]$ only if the linearized system (7) is admissible for arbitrary symmetric matrix $K_{\Delta}$ with $0<=$ $K_{\Delta}<=K$.

REMARK 4. By Theorem 1, the admissibility of $(E, A)$ is a necessary condition for LDS (5) to be strongly absolutely stable with respect to $F[0, K]$, so we can safely assume that $(E, A)$ is admissible.

Since $(E, A)$ is admissible, there exist two nonsingular matrices $M, N \in R^{n \times n}$, such that

$$
M E N=\left[\begin{array}{cc}
I_{r} & 0 \\
0 & 0
\end{array}\right], \quad M A N=\left[\begin{array}{cc}
A_{1} & 0 \\
0 & I
\end{array}\right]
$$

where $A_{1} \in R^{r \times r}$. Compatible with (8), partition $M B$ and $C N$ as follows

$$
M B=\left[\begin{array}{c}
B_{1} \\
B_{2}
\end{array}\right], \quad C N=\left[\begin{array}{ll}
C_{1} & C_{2}
\end{array}\right] .
$$

And let

$$
N^{-1} x=\left[\begin{array}{l}
x_{1} \\
x_{2}
\end{array}\right]
$$

Thus LDS (5) is transformed to

$$
\begin{aligned}
& \dot{x}_{1}=A_{1} x_{1}+B_{1} \omega, \\
& x_{2}=-B_{2} u, \\
& \sigma=C_{1} x_{1}+\left(D-C_{2} B_{2}\right) \omega, \\
& \omega=-\phi(\sigma) .
\end{aligned}
$$


It is easy to see that the strongly absolutely stability of LDS (5) is equivalent to that of system (10).

Consider the LSS

$$
\begin{aligned}
& \dot{x}_{1}=A_{1} x_{1}+B_{1} \omega, \\
& \sigma=C_{1} x_{1}+\left(D-C_{2} B_{2}\right) \omega, \\
& \omega=-\phi(\sigma),
\end{aligned}
$$

which is obtained from (10) by removing the second equation. We shall discuss the relationship between (10) and (11), by which we investigate the strongly absolute stability of LDS (5). To do this, the following lemma is useful.

Lemma 1 (Liao, 1993). The identity

$$
\operatorname{det}(I+G H)=\operatorname{det}(I+H G)
$$

holds for arbitrary matrices $H$ and $G$ as long as $G H$ and $H G$ exist. The idenity matrices on both sides can be different of order.

Theorem 2. LDS (5) is strongly absolutely stable if and only if system (11) is absolutely stable.

Proof. Necessity is obvious. We only prove the sufficiency.

Assume that system (11) is absolutely stable.

Set $\phi(\sigma)=K_{\Delta} \sigma$, where $K_{\Delta}$ is an arbitrary diagonal matrix with $0<=K_{\Delta}<=K$, system (11) reduces to

$$
\begin{aligned}
& \dot{x}_{1}=A_{1} x_{1}-B_{1} K_{\Delta} \sigma \\
& \sigma=C_{1} x_{1}-\left(D-C_{2} B_{2}\right) K_{\Delta} \sigma
\end{aligned}
$$

which is asymptotically stable. Thus, it is necessary that $I+\left(D-C_{2} B_{2}\right) K_{\Delta}$ is nonsingular, that is

$$
\operatorname{det}\left(I+\left(D-C_{2} B_{2}\right) K_{\Delta}\right) \neq 0
$$

If it is not the case, system (12) is degenerate and represents an unstable system (Vidyasagar, 1978).

Let $F(\sigma)=\sigma+\left(D-C_{2} B_{2}\right) \phi(\sigma)$, it is obvious that $F(\sigma)$ is continuous and $F(0)=0$. Assume there exists $\sigma_{0} \neq 0$ satisfying $F\left(\sigma_{0}\right)=0$, then $\sigma_{0}=-\left(D-C_{2} B_{2}\right) \phi\left(\sigma_{0}\right)$. Since $\phi \in F[0, K]$, there exists $K_{\Delta 0}$ with $0<=K_{\Delta 0}<=K$ such that $\phi\left(\sigma_{0}\right)=K_{\Delta 0} \sigma_{0}$. Then $\sigma_{0}=-\left(D-C_{2} B_{2}\right) K_{\Delta 0} \sigma_{0}$ which indicates $I+\left(D-C_{2} B_{2}\right) K_{\Delta 0}$ is singular, as contradicts with (13). Thus we can claim that $F(\sigma)=0$ has unique solution $\sigma=0$. 
By (11), $F(\sigma)=C_{1} x_{1}$, then the absolute stability of (11) implies that

$$
\lim _{t \rightarrow+\infty} F(\sigma)=0
$$

thus

$$
\lim _{t \rightarrow+\infty} \sigma=0
$$

which yields

$$
\lim _{t \rightarrow+\infty} \phi(\sigma)=0
$$

Then, considering system (10)

$$
\lim _{t \rightarrow+\infty} x_{2}(t)=\lim _{t \rightarrow+\infty} B_{2} \phi(t)=0
$$

that is, system (10) is globally asymptotically stable.

Thus $x_{1}=0$ implies $x_{2}=0$ and $\sigma=0$.

Now we will prove system (10) is impulsive-free for all $\phi \in F[0, K]$.

Rewrite system (10) in the following form

$$
\left[\begin{array}{cc}
I_{r} & 0 \\
0 & 0
\end{array}\right]\left[\begin{array}{c}
\dot{x}_{1} \\
\dot{x}_{2}
\end{array}\right]=\left[\begin{array}{c}
F_{1}\left(x_{1}, x_{2}\right) \\
F_{2}\left(x_{1}, x_{2}\right)
\end{array}\right],
$$

where $F_{1}\left(x_{1}, x_{2}\right)=A_{1} x_{1}-B_{1} \phi(\sigma), F_{2}\left(x_{1}, x_{2}\right)=x_{2}-B_{2} \phi(\sigma)$.

Then,

$$
\begin{aligned}
& \partial F_{2} /\left.\partial x_{2}\right|_{x_{1}=0, x_{2}=0}=I-B_{2} \partial \phi /\left.\partial x_{2}\right|_{x_{1}=0, x_{2}=0} \\
& \quad=I-\left.B_{2}(\partial \phi / \partial \sigma)\left(\partial \sigma / \partial x_{2}\right)\right|_{x_{1}=0, x_{2}=0} .
\end{aligned}
$$

By the third equation of (10),

$$
\partial \sigma /\left.\partial x_{2}\right|_{x_{1}=0, x_{2}=0}=\left(I+\partial \phi /\left.\partial \sigma\right|_{x_{1}=0, x_{2}=0} D\right)^{-1} \partial \phi /\left.\partial \sigma\right|_{x_{1}=0, x_{2}=0} C_{2}
$$

which together with (17) gives

$$
\begin{aligned}
& \partial F_{2} /\left.\partial x_{2}\right|_{x_{1}=0, x_{2}=0}=I-B_{2}\left(I+\partial \phi /\left.\partial \sigma\right|_{x_{1}=0, x_{2}=0} D\right)^{-1} \partial \phi /\left.\partial \sigma\right|_{x_{1}=0, x_{2}=0} C_{2} \\
& \quad=I-B_{2}\left(I+\partial \phi /\left.\partial \sigma\right|_{\sigma=0} D\right)^{-1} \partial \phi /\left.\partial \sigma\right|_{\sigma=0} C_{2} .
\end{aligned}
$$

Since $\phi \in F[0, K]$, there exists $K_{\Delta}$ with $0<=K_{\Delta}<=K$ such that $\partial \phi /\left.\partial \sigma\right|_{\sigma=0}=K_{\Delta}$, the well-poseness of (5) indicates that the inverse $\left(I+K_{\Delta} D\right)^{-1}$ does exist and Lemma 1 together with (13) show that $\partial F_{2} /\left.\partial x_{2}\right|_{x_{1}=0, x_{2}=0}$ is nonsingular, so is $\partial F_{2} / \partial x_{2}$ around the point $x_{1}=0, x_{2}=0$ by the continuity of $\partial \phi / \partial \sigma$. Consequently, system (10) is impulsive-free. Hence, system (10) is strongly absolutely stable with respect to $F[0, K]$. So is LDS (5). 
Consider the generalized Lyapunov function (Ishihara and Terra, 2002)

$$
V(x)=x^{T} E^{T} P x,
$$

where $P \in R^{n \times n}$ satisfies $E^{T} P=P^{T} E>=0$ with $\operatorname{rank}\left(E^{T} P\right)=r$.

The following lemma will be used in the sequel.

Lemma 2 (Boyd and Ghaoui, 1994). (S-procedure)

Let $T_{0}, T_{1}, \cdots, T_{p} \in R^{n \times n}$ be symmetric matrices. The following condition on $T_{0}, T_{1}, \cdots, T_{p}$ :

$$
\zeta^{T} T_{0} \zeta>0, \quad \forall \zeta \neq 0, \quad \zeta^{T} T_{i} \zeta>=0, \quad i=1, \cdots, p
$$

holds if and only if there exist $\tau_{i}>=0$ such that

$$
T_{0}-\sum_{i=1}^{p} T_{i}>0 .
$$

It is a nontrival fact that for $p=1$, the converse holds if there is some $\zeta_{0}$ such that $\zeta_{0}^{T} T_{1} \zeta_{0}>0$

Theorem 3. The following statements are equivalent and guarantee the strongly absolute stability of $L D S$ (5)

i) For $\forall x \neq 0$ satisfying constraint (6)

$$
\left.\dot{V}(x)\right|_{(5)}<0 ;
$$

ii) there exists matrix $P$ and scalar $\tau>0$ such that

$$
\begin{aligned}
& {\left[\begin{array}{ll}
A^{T} P+P^{T} A & \tau C^{T} K-P^{T} B \\
\tau K C-B^{T} P & -\tau\left(2 I+K D+D^{T} K\right)
\end{array}\right]<0,} \\
& E^{T} P=P^{T} E>=0 .
\end{aligned}
$$

Proof. We first prove the equivalency between i) and ii).

LMI (21) indicates that $P$ is nonsingular, consequently, $\operatorname{rank}\left(E^{T} P\right)=\operatorname{rank}(E)=r$.

Calculating the derivative of $V(x)$ along the solution of LDS (5) gives

$$
\begin{aligned}
\left.\dot{V}(x)\right|_{(5)} & =\dot{x}^{T} E^{T} P x+x^{T} E^{T} P \dot{x} \\
& =x^{T}\left(A^{T} P+P^{T} A\right) x-2 x^{T} P^{T} B \phi .
\end{aligned}
$$

Thus, by Lemma 2, i) holds if and only if there exists $\tau>=0$ such that (21) holds. And $\tau=0$ is impossible by (21).

Next, we prove ii) implies that LDS (5) is strongly absolutely stable. 
Assume that ii) holds. It is easy to show that $(E, A)$ is admissible. Without loss of generality, we assume that LDS (5) is in the form of (10). Then, by Theorem 2, we can consider LSS (11) to investigate the strongly absolute stability of LDS (5).

Partition

$$
P=\left[\begin{array}{ll}
P_{11} & P_{12} \\
P_{21} & P_{22}
\end{array}\right]
$$

conformably to (10), then we have $P_{11}=P_{11}^{T}>=0, P_{12}=0$ in view of $E^{T} P=$ $P^{T} E>=0$.

Then LMI (21) can be written as

$$
\left[\begin{array}{ccc}
A_{1}^{T} P_{11}+P_{11} A_{1} & P_{21}^{T} & (1,3) \\
P_{21} & P_{22}+P_{22}^{T} & \tau C_{2}^{T} K-P_{22}^{T} B_{2} \\
(1,3)^{T} & \tau K C_{2}-B_{2}^{T} P_{22} & -\tau\left(2 I+K D+D^{T} K\right)
\end{array}\right]<0,
$$

where $(1,3)=\tau C_{1}^{T} K-P_{11} B_{1}-P_{21}^{T} B_{2}$.

Pre-multiplying and post-multiplying (23) by

$$
\left[\begin{array}{ccc}
I & 0 & 0 \\
0 & B_{2}^{T} & I \\
0 & I & 0
\end{array}\right]
$$

and it's transposition respectively gives

$$
\left[\begin{array}{ccc}
A_{1}^{T} P_{11}+P_{11} A_{1} & \tau C_{1}^{T} K-P_{11} B_{1} & P_{21}^{T} \\
\tau K C_{1}-B_{1}^{T} P_{11} & (2,2) & B_{2}^{T} P_{22}^{T}+\tau K C_{2} \\
P_{21} & P_{22} B_{2}+\tau C_{2}^{T} K & P_{22}+P_{22}^{T}
\end{array}\right]<0,
$$

which implies

$$
\left[\begin{array}{cc}
A_{1}^{T} P_{11}+P_{11} A_{1} & \tau C_{1}^{T} K-P_{11} B_{1} \\
\tau K C_{1}-B_{1}^{T} P_{11} & (2,2)
\end{array}\right]:=S<0
$$

where $(2,2)=-\tau\left(2 I+K D+D^{T} K-K C_{2} B_{2}-B_{2}^{T} C_{2}^{T} K\right)$.

Let $V\left(x_{1}\right)=x_{1}^{T} P_{11} x_{1}$, and calculate the derivative of $V\left(x_{1}\right)$ along the trajectory of system (11), we have

$$
\begin{aligned}
\dot{V}( & \left.x_{1}\right)\left.\right|_{(11)}=\dot{x}_{1}^{T} P_{11} x_{1}+x_{1}^{T} P_{11} \dot{x}_{1} \\
= & \left(A_{1} x_{1}+B_{1} \omega\right)^{T} P_{11} x_{1}+x_{1}^{T} P_{11}\left(A_{1} x_{1}+B_{1} \omega\right) \\
= & x_{1}^{T}\left(A_{1}^{T} P_{11}+P_{11} A_{1}\right) x_{1}+2 x_{1}^{T} P_{11} B_{1} \omega-2 \tau \phi^{T}(\phi-K \sigma)+2 \tau \phi^{T}(\phi-K \sigma) \\
= & x_{1}^{T}\left(A_{1}^{T} P_{11}+P_{11} A_{1}\right) x_{1}-2 x_{1}^{T} P_{11} B_{1} \phi \\
& -\tau \phi^{T}\left(2 I+K D+D^{T} K\right) \phi+2 \tau \phi^{T}\left(K C_{1} x_{1}+K C_{2} B_{2} \phi\right)+2 \tau \phi^{T}(\phi-K \sigma)
\end{aligned}
$$




$$
\begin{aligned}
= & x_{1}^{T}\left(A_{1}^{T} P_{11}+P_{11} A_{1}\right) x_{1}+2 x_{1}^{T}\left(C_{1}^{T}-P_{11} B_{1}\right) \phi \\
& -\tau \phi^{T}\left(2 I+K D+D^{T} K-K C_{2} B_{2}-B_{2}^{T} C_{2}^{T} K\right) \phi+2 \tau \phi^{T}(\phi-K \sigma) \\
= & {\left[\begin{array}{ll}
x_{1}^{T} & \phi^{T}
\end{array}\right] S\left[\begin{array}{c}
x_{1} \\
\phi
\end{array}\right]+2 \tau \phi^{T}(\phi-K \sigma) . }
\end{aligned}
$$

In view of (25) and (6), (26) implies

$$
\left.\dot{V}\left(x_{1}\right)\right|_{(11)}<0
$$

for any $x_{1} \neq 0$ satisfying constraint (6), then $\lim _{t \rightarrow+\infty} x_{1}(t)=0$, which yields that LSS(11) is absolutely stable. Then, by Theorem 2, LDS (5) is strongly absolutely stable.

REMARK 5. Note that we restrict the nonlinearities $\phi$ to be time-invariant in the above definitions and results. If $\phi$ is time-varying, the notion of strongly absolute stability of LDS (5) can be defined analogously to Definition 3, however, Theorem 2 may not hold any more. But condition ii) of Theorem 3 guarantees LDS (5) is strongly absolutely stable even though $\phi$ is time-varying. To show this, assume that condition ii) of Theorem 3 holds. Then, by the proof of Theorem 3, we can conclude that LSS (11) is strongly absolutely stable and

$$
2 I+K D+D^{T} K-K C_{2} B_{2}-B_{2}^{T} C_{2}^{T} K>0
$$

Following the proof of Theorem 2, let

$$
F=\sigma+\left(D-C_{2} B_{2}\right) \phi(\sigma, t)
$$

then (14) holds. Furthermore, in view of (6), there exists $\alpha>0$, such that

$$
\begin{aligned}
2 \phi^{T} K F & =2 \phi^{T} K \sigma+2 \phi^{T} K\left(D-C_{2} B_{2}\right) \phi \\
> & =2 \phi^{T} \phi+2 \phi^{T} K\left(D-C_{2} B_{2}\right) \phi \\
> & =\alpha \phi^{T} \phi .
\end{aligned}
$$

On the other hand, for any $\gamma>0$,

$$
2 \phi^{T} K F<=\gamma F^{T} K K F+\gamma^{-1} \phi^{T} \phi
$$

then we can choose some $\gamma>0$ such that $\alpha-\gamma^{-1}>0$ satisfying

$$
\gamma F^{T} K K F>=\left(\alpha-\gamma^{-1}\right) \phi^{T} \phi
$$

then (14) implies that (15). Continuing to use the proof for Theorem 2, it is easy to validate that condition ii) of Theorem 3 guarantees strongly absolute stability of LDS (5) even if $\phi$ is time-varying. 
Now, we will present frequency-domain interpretation for the above LMIs based criterion. To achieve this, the following lemma is required.

Lemma 3 (Zhang et al., 2002). The following statements are equivalent.

1) $(E, A)$ is admissible, $D+D^{T}>0$ and $G(s)$ is ESPR.

2) The following LMIs are feasible

$$
\begin{aligned}
& {\left[\begin{array}{cc}
A^{T} X+X^{T} A & C^{T}-X^{T} B \\
C-B^{T} X & -\left(D+D^{T}\right)
\end{array}\right]<0,} \\
& E^{T} X=X^{T} E>=0,
\end{aligned}
$$

where $G(s)=C(s E-A)^{-1} B+D$.

COROllary 1. Condition ii) of Theorem 3 holds if and only if

$$
I+K G(s)
$$

is ESPR and $2 I+K D+D^{T} K$ is positive definite.

Proof. By simple computation, we have

$$
\tau(I+K D)+\tau K C(s E-A)^{-1} B=\tau(I+K G(s)) .
$$

Then by Lemma 3 and under the assumption that $(E, A)$ is admissible, condition ii) of Theorem 3 holds if and only if $\tau(I+K G(s))$ is ESPR and $2 I+K D+D^{T} K$ is positive definite. At the same time, it is evident that for any $\tau>0, \tau(I+K G(s))$ is ESPR if and only if $I+K G(s)$ is ESPR, thus we complete the proof.

REMARK 6. Corollary 1 is a generalization of the classical circle criterion.

Corollary 1 indicates that the variable $\tau$ in LMI (21) is not necessary for the LMI feasibility problem of Theorem 3 and we can set it to be any fixed positive real number, for example, $\tau=1$. However, when one deals with multiple objects analysis problem of LDS (5), the variable $\tau$ is useful and can reduce the conservatism. To see this, we consider the following uncertain LDS

$$
\begin{aligned}
& E \dot{x}=(A+\Delta A) x+(B+\Delta B) w, \\
& \sigma=C x+D w \\
& w=-\phi(t, \sigma)
\end{aligned}
$$

where $\Delta A$ and $\Delta B$ are time-invariant matrix representing norm-bounded parameter uncertainty and assumed to be of the form

$$
\begin{aligned}
& {\left[\begin{array}{ll}
\Delta A & \Delta B
\end{array}\right]=H F(\theta)\left[\begin{array}{ll}
E_{1} & E_{2}
\end{array}\right],} \\
& F^{T}(\theta) F(\theta)<=I, \quad \forall \theta \in \Xi,
\end{aligned}
$$


$\theta \in \Xi$, where $\Xi$ is a compact set. The matrix $H, E_{1}, E_{2}$ are known.

Theorem 4. Uncertain LDS (30) is robustly strongly absolutely stable with respect to $F[0, K]$ if there exists $P, \tau>0$ and $\varepsilon>0$, such that

$$
\begin{aligned}
& {\left[\begin{array}{ccc}
A^{T} P+P^{T} A+E_{1}^{T} E_{1} & \tau C^{T} K-P^{T} B+E_{1}^{T} E_{2} & P^{T} H \\
\tau K C-B^{T} P+E_{2}^{T} E_{1} & -\tau\left(K D+D^{T} K\right)-2 \tau I+E_{2}^{T} E_{2} & 0 \\
H^{T} P & 0 & -\varepsilon I
\end{array}\right]<0,} \\
& E^{T} P=P^{T} E>=0 .
\end{aligned}
$$

Proof. By Theorem 3, system (30) is strongly absolutely stable with respect to $F[0, K]$ if there $P$, such that

$$
\begin{aligned}
& {\left[\begin{array}{cc}
(A+\Delta A)^{T} P+P^{T}(A+\Delta A) & \tau C^{T} K-P^{T}(B+\Delta B) \\
\tau K C-(B+\Delta B)^{T} P & -\tau\left(K D+D^{T} K\right)-2 \tau I
\end{array}\right]<0} \\
& E^{T} P=P^{T} E>=0
\end{aligned}
$$

Denote

$$
\begin{aligned}
& Y_{0}=\left[\begin{array}{cc}
A^{T} P+P^{T} A & \tau C^{T} K-P^{T} B \\
\tau K C-B^{T} P & -\tau\left(K D+D^{T} K\right)-2 \tau I
\end{array}\right], \\
& Y=\left[\begin{array}{cc}
P^{T} \Delta A & -P^{T} \Delta B \\
0 & 0
\end{array}\right] .
\end{aligned}
$$

By (31), we have

$$
\begin{aligned}
Y(t) & =\left[\begin{array}{cc}
P^{T}\left(H F(\theta) E_{1}\right) & -P^{T}\left(H F(\theta) E_{2}\right) \\
0 & 0
\end{array}\right] \\
& =\left[\begin{array}{c}
P^{T} H \\
0
\end{array}\right] F(\theta)\left[\begin{array}{ll}
E_{1} & E_{2}
\end{array}\right] .
\end{aligned}
$$

Thus, using the routine method of handling norm bounded uncertainties (Xie, 1996), there exists $\varepsilon>0$ such that

$$
Y_{0}+\varepsilon\left[\begin{array}{c}
P^{T} H \\
0
\end{array}\right]\left[\begin{array}{c}
P^{T} H \\
0
\end{array}\right]^{T}+\varepsilon^{-1}\left[\begin{array}{ll}
E_{1} & E_{2}
\end{array}\right]^{T}\left[\begin{array}{ll}
E_{1} & E_{2}
\end{array}\right]<0
$$

which is equivalent to (33).

\section{Numerical Examples}

In this section, numerical examples are given to illustrate our results. Matlab 6.5 is used to check the LMIs feasibility problem. To deal with the non-strict LMI (22), let $E_{0} \in$ 
$R^{n \times(n-r)}$ be a matrix of full-column rank such that $E^{T} E_{0}=0$, and $r=\operatorname{rankE}$. We introduce two new matrix variables $X \in R^{n \times n}$ and $Q \in R^{(n-r) \times n}$ and assume that $P=X E+E_{0} Q$. Then it easy to show that $E^{T} P=E^{T} X E=P^{T} E>=0$ if $X$ is symmetric and positive definite. Then, by the statements in (Ishihara and Terra, 2002), we have the following to corollaries.

Corollary 2. Condition ii) of Theorem 3 holds if and only if there exist $X \in R^{n \times n}$ with $X>0, Q \in R^{(n-r) \times n}$, and $\tau>0$ such that

$$
\left[\begin{array}{cc}
A^{T}\left(X E+E_{0} Q\right)+\left(X E+E_{0} Q\right)^{T} A & \tau C^{T} K-\left(X E+E_{0} Q\right)^{T} B \\
\tau K C-B^{T}\left(X E+E_{0} Q\right) & -2 I-K D-D^{T} K
\end{array}\right]<0 .
$$

COROLlary 3. Conditions of Theorem 4 hold if and only if there exist $X \in R^{n \times n}$ with $X>0, Q \in R^{(n-r) \times n}, \tau>0$ and $\varepsilon>0$ such that

$$
\left[\begin{array}{ccc}
(1,1) & (1,2) & \left(X E+E_{0} Q\right)^{T} H \\
(1,2)^{T} & (2,2) & 0 \\
H^{T}\left(X E+E_{0} Q\right) & 0 & -\varepsilon I
\end{array}\right]<0,
$$

where $(1,1)=A^{T}\left(X E+E_{0} Q\right)+\left(X E+E_{0} Q\right)^{T} A+E_{1}^{T} E_{1}$ and $(1,2)=\tau C^{T} K-$ $\left(X E+E_{0} Q\right)^{T} B+E_{1}^{T} E_{2},(2,2)=-\tau\left(K D+D^{T} K\right)-2 \tau I+E_{2} E_{2}$.

EXAMPLE 1. Consider a descriptor system with system matrices

$$
\begin{aligned}
& E=\left[\begin{array}{llll}
2 & 0 & 0 & 0 \\
0 & 1 & 2 & 0 \\
1 & 0 & 1 & 0 \\
0 & 0 & 0 & 0
\end{array}\right], \quad A=\left[\begin{array}{cccc}
-10 & 0 & 4 & 0 \\
0 & -10 & 2 & 0 \\
0 & 0 & -4 & 1 \\
0 & 2 & 0 & 1
\end{array}\right], \\
& C=\left[\begin{array}{llll}
0 & 1 & 1 & 0 \\
1 & 1 & 4 & 0
\end{array}\right], \quad B^{T}=\left[\begin{array}{cccc}
1 & 1 & 0 & 0 \\
0 & 1 & 0 & -1
\end{array}\right], \\
& K=\left[\begin{array}{ll}
2 & 1 \\
1 & 1
\end{array}\right], \quad D=\left[\begin{array}{cc}
0 & 0 \\
0 & 0.5
\end{array}\right] .
\end{aligned}
$$

Let

$$
E_{0}=\left[\begin{array}{llll}
0 & 0 & 0 & 1
\end{array}\right]^{T} .
$$

Solving LMI (39) gives

$$
\begin{aligned}
X & =\left[\begin{array}{rrrr}
0.0662 & 0.0324 & -0.0988 & 0.0000 \\
0.0324 & 0.0977 & -0.1062 & 0.0000 \\
-0.0988 & -0.1062 & 0.3294 & -0.0000 \\
0.0000 & 0.0000 & -0.0000 & 0.5443
\end{array}\right], \\
Q & =\left[\begin{array}{llll}
-0.0777 & 0.2074 & -0.0563 & -0.0739
\end{array}\right], \quad \tau=0.0530 .
\end{aligned}
$$




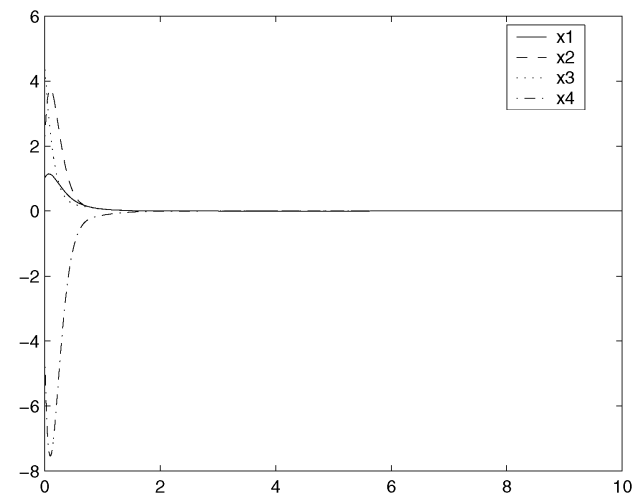

Fig. 1. State responses with $x(0)=[1,2,4.6,-4]^{T}$.

So this system is strongly absolutely stable with respect to $F[0, K]$. In addition, LMI (39) is also feasible if we set $\tau=1$.

Let $\phi(t, \sigma)=\left[\begin{array}{l}\phi_{1} \\ \phi_{2}\end{array}\right]$, where $\phi_{1}=0.5 *\left(2 \sigma_{1}+\sigma_{2}+\sin \left(2 \sigma_{1}+\sigma_{2}\right)\right), \phi_{2}=\sin ^{2}(t)\left(\sigma_{1}+\sigma_{2}\right)$ and Fig. 1 shows the state response of the system.

EXAmPLE 2. Consider the uncertain LDS (30) with the system matrices $A, B, C, D$ are the same as those given in Example 1. The uncertainties are represented in the form of (31) with

$$
H=\left[\begin{array}{ll}
1 & 0 \\
0 & 1 \\
0 & 1 \\
0 & 0
\end{array}\right], \quad E_{1}=\left[\begin{array}{llll}
1 & 0 & 0 & 0 \\
0 & 1 & 0 & 0
\end{array}\right], \quad E_{2}=\left[\begin{array}{ll}
1 & 0 \\
0 & 1
\end{array}\right] .
$$

Solving LMIs in Corollary 3 yields

$$
\begin{aligned}
& X=\left[\begin{array}{rrrr}
0.3459 & 0.1498 & -0.4934 & 0.0000 \\
0.1498 & 0.4920 & -0.5555 & 0.0000 \\
-0.4934 & -0.5555 & 1.9239 & -0.0000 \\
0.0000 & 0.0000 & -0.0000 & 2.3088
\end{array}\right] \times 10^{3}, \\
& Q=\left[\begin{array}{lll}
-0.5664 & 1.1087-0.4541 & -0.3097
\end{array}\right] \times 10^{3}, \\
& \tau=301.9121, \quad \varepsilon=2.3454 \times 10^{3} .
\end{aligned}
$$

So the system is robustly strongly absolutely stable. At the same time, if we set $\tau=1$, the LMIs are found to be not feasible. This also demonstrates that the variable $\tau$ results in less conservative result if there exist uncertainties in the system matrices. 


\section{Conclusions}

In this paper, we consider Lur'e type descriptor systems(LDS) and introduced a new stability concept - strongly absolute stability for LDS. Such a notion is a generalization of absolute stability for LSS and admissibility of linear time-invariant descriptor systems. Following the methodologies of absolute stability of LSS, a linearized system of LDS is introduced to derive a necessary condition on strongly absolute stability. A reducedorder LSS is obtained by a standard coordinate transformation, and the strongly absolute stability of the LDS is proved to be equivalent to the absolute stability of the LSS. The obtained stability criterion can be view as a generalization of the classical circle criterion for LSS. Finally, numerical examples illustrate our results.

\section{Acknowledgments}

This work was supported by the Natural Science Foundation of China under Grant No. 60574011.

\section{References}

Boyd, S., and L.E. Ghaoui (1994). Linear Matrix Inequalities in System and Control Theory. SIAM, Philadelphia, PA.

Brenan, K.E., S.L. Campbell and L.R. Petzold (1996). Numerical Solution of Initial-Value Problems in Differential-Algebraic Equations. SIAM, Philadelphia.

Campbell, S.L. (1980). Singular Systems of Differential Equations. Pitman, London.

Dai, L. (1989). Singular Control Systems. Springer, Berlin, Germany.

Haddad, W.M., and D.S. Bernstein (1993). Explicit construction of quadratic Lyapunov functions for the small gain, positivity, circle and Popov theorems and their application to robust stability part i: continous-time theory. Int. J. Robust and Nonlinear Control, 3(4), 313-339.

Haddad, W.M., and D.S. Bernstein (1994). Explicit construction of quadratic Lyapunov functions for the small gain, positivity, circle and Popov theorems and their application to robust stability part ii: Discrete-time theory. Int. J. Robust and Nonlinear Control, 4(2), 249-265.

Ishihara, J.Y., and M.H. Terra (2002). On the Lyapunov theorem for singular systems. IEEE Trans. Automat. Contr., 47(11), 1926-1930.

Khalil, H.K. (1996). Nonlinear Systems. Third Edition. Prentice Hall.

Lee, L., and J.L. Chen (2003). Strictly positive real lemma and absolute stability for discrete-time descriptor systems. IEEE Trans. Circuits and Systems I, 50(6), 788-794.

Lewis, E.L. (1986). A survey of linear singular systems. Circuits, Systems and Signal Processing, 5(1), 3-36.

Li, Y.Q., and Y.Q. Liu (1998). Stability of solutions of singular systems with delay. Control Theory and Applications, 15(4), 542-550.

Liao, X. (1993). Absolute Stability of Nonlinear Control Systems. Science Press, Beijing.

Mohler, R.R. (1991). Nonlinear Systems. Prentice Hall, Englewood Cliffs, New Jersey.

Muller, P.C. (1997). Linear mechanical descriptor systems: identification, analysis and design. In Preprints of IFAC. Conference on Control of Industrial Systems. Belfort, France. pp. 501-506.

Silva, M.S., and T.P. De Lima (2003). Looking for nonnegative solutions of a leontief dynamic model. Linear Algebra and its Applications, 364(1), 281-316.

Sun, W., P.P. Khargonekar and D. Shim (1994). Solution to the positive real control problem for linear timeinvariant systems. IEEE Trans. Automat. Contr, 39(10), 2034-2046.

Vidyasagar, M. (1978). Nonlinear Systems Analysis. Prentice Hall, Englewood Cliffs, New Jersey. 
Vladimir, B.B. (1986). Partial stability of motion of semi-state systems. Int. J. Control, 44(5), 1383-1394.

Vladimir, B.B. (1987). Lyapunov function candidates for semi-state systems. Int. J. Control, 46(6), 2171-2181.

Vladimir, B.B., and M.M. Mirko (1987). Extended stability of motion of semi-state systems. Int. J. Control, 46(6), 2183-2197.

Wu, H., C. Yung and F. Chang (2002). $H_{\infty}$ control for nonlinear descriptor systems. IEEE Trans. Automat. Contr., 47(11), 1919-1925.

Wu, H., and K. Mizukami (1995). Lyapunov stability theory and robust control of uncertain descriptor systems. Int. J.System Sci., 26(10), 1981-1991.

Xie, L. (1996). Output feedback $H_{\infty}$ control of systems with parameter uncertainty. Int. J. Control, 63(4), 741-750.

Zhang, L., J. Lam and S. Xu (2002). On positive realness of descriptor systems. IEEE Trans. Circiuts and Systems I, 49(3), 401-407.

C. Yang received BSc degree from the Mathematics Department Northeastern University, Shenyang, China in 2002. He is now a PhD candidate at Northeastern University. And his research interests include descriptor systems, robust control and fuzzy control.

Q. Zhang received the BSc and MSc degrees from the Mathematics Department and the $\mathrm{PhD}$ degree from the Automatic Control Department of Northeastern University, Shenyang, China, in 1982, 1986, and 1995, respectively. He finished his two-year postdoctoral work in Automatic Control Department of Northwestern Polytechnical University, Xi'an, China, in 1997. Since then, he has been a professor and dean of the College of Science at Northeastern University. He is also a member of the University Teaching Advisory Committee of National Ministry of Education. He has published six books and more than 230 papers about control theory and applications. Dr. Zhang received 14 prizes from central and local governments for his research. He has also received the Golden Scholarship from Australia in 2000. During these periods, he visited Hong Kong University, Sydney University, Western Australia University and Niigata University, Pohan University of Science and Technology, Seoul University, Alberta University, Lakehead University and Wisor University as a Research Associate, Research Fellow, Senior Research Fellow and visiting professor, respectively.

L. Zhou received BSc degree from the Department of Water Conservancy Engineering North China Institute of Water Conservancy and Hydroelectric Power, Zhengzhou, China in 2001. She is now a PhD candidate at Northeastern University. And his research interests include descriptor systems, robust control and fuzzy control.

\title{
Deskriptorinių sistemų griežto absoliutinio stabilumo problema
}

\author{
Chunyu YANG, Qingling ZHANG, Linna ZHOU
}

Straipsnyje nagrinejjamos Lur tipo deskriptorinès sistemos (LDS). Apibrěžiama griežto absoliutinio stabilumo sąlyga ir teigiama, kad tai yra standartinių Lur tipo būsenų erdvès sistemų (LSS) apibendrinimas. LDS eilè yra sumažinama transformuojant koordinates. Parodyta, kad LDS griežtas absoliutinis stabilumas ekvivalentus žemesnès eilès LSS absoliutiniam stabilumui. Panaudota apibendrintoji Liapunovo funkcija ir išvestas griežtas absoliutinis stabilumo kriterijus. Paaiškinama šio kriterijaus prasmė dažnių srityje. Pateiktas skaitmeninis pavyzdys, iliustruojantis gautų teorinių rezultatų efektyvumą. 